\title{
Conocer la realidad estatal. Arturo Sampay, lector de Hermann Heller.
}

Knowing the State's Reality. Arturo Sampay, Reader of Hermann Heller

\author{
Por: Fraile, Nicolás* \\ IIGG-UBA, CONICET
}

Ciudad Autónoma de Buenos Aires, Argentina.

Email: nicolas.fraile@gmail.com

Fecha de recepción: 31/08/2020

Fecha de aprobación: 09/09/2020

DOI: http://dx.doi.org/10.30972/nvt.1624494

\section{Resumen}

El propósito de este artículo es indagar el papel que cumple Hermann Heller en la obra de Arturo Sampay, principalmente en lo relativo a la epistemología y metodología de la teoría del Estado. El artículo se divide en dos apartados. En el primero indagamos la utilización que Sampay hace de Heller para someter a crítica lo que denomina la teoría idealista del Estado, esto es, aquella que concibe a la realidad estatal como una formación abstracta, y para fundamentar el carácter real y objetivo de la estatalidad. En el segundo, restituimos la utilización que Sampay hizo de Heller para desarrollar una comprensión propia de la teoría del Estado, tal como hizo en la segunda parte de su obra de 1951 titulada Introducción a la teoría del Estado.

Palabras clave: Sampay - Heller - teoría del Estado - teoría idealista metodología.

\footnotetext{
* Licenciado en Ciencia Política por la Facultad de Ciencias Sociales (UBA) y maestrando en Teoría Política y Social por la misma facultad. Becario doctoral de CONICET con sede de trabajo en el Instituto de Investigaciones Gino Germani (FSOC-UBA).
} 


\section{Nicolás Fraile}

\section{Abstract}

The aim of this article is to analize the rol that plays Hermann Heller in the work of Arturo Sampay, mainly in the epistemology and methodology of the theory of the State. According to this aim, the article is divided into two sections. In the first section, we analyze the use Sampay makes of Heller to criticize the idealist theory of the State, i.e., the understanding of the State's reality as an abstract form, and to found its objective and real character. In the second one, we follow the use of Heller in order to develop his own comprehension of this discipline, as Sampay did in the second part of his work in 1951 called Introduction to the theory of the State.

Keywords: Sampay - Heller - Theory of the State - Idealist Theory Methodology.

\section{Cómo citar este artículo:}

APA: Fraile, N. (2020). Conocer la realidad estatal. Arturo Sampay, lector de Hermann Heller. Nuevo Itinerario, 16 (2), 102-126. Recuperado de: (agregar dirección web)

\section{Introducción}

La obra de Hermann Heller no solo ha tenido un significativo influjo en la teoría política y el pensamiento jurídico de la República de Weimar (Jacobson y Schlink, 2001; Llanque, 2019) o en las ciencias sociales de la República Federal Alemana, donde fue señalado como "padre de la ciencia política" (Mommsen, 1962: 350), sino también en la teoría política argentina: para el entrerriano Arturo Sampay, íntimamente vinculado al peronismo y a la Constitución sancionada en 1949, la obra de Heller supuso uno de los más importantes insumos para la reflexión teórica en obras como La crisis del Estado de derecho liberal-burgués [1943] o, principalmente, en su Introducción a la Teoría del Estado [1951]. El objetivo de este artículo es indagar en la lectura que Sampay hizo de Heller a fin de conocer el lugar y papel que desempeña en su obra.

El vínculo entre Sampay y Heller parece tener un primer asiento de carácter biográfico. Tras graduarse en 1932, el entrerriano emprendió un viaje por Europa que lo llevó a tomar un curso de derecho público en la ciudad de Zúrich con un discípulo directo de Heller: Dietrich Schindler (Gómez Arboleya, 1963: 147; Segovia, 2007: 172). 
El vasto conocimiento que Sampay tenía de la obra de Heller, entonces, puede provenir de estas lecciones con Schindler durante su viaje por Europa, en el que también conoció y estudió con profesores como Monseñor Olgiati o Jacques Maritain.

Sin embargo, a nivel teórico, el vínculo que Sampay tendió con Heller excede ampliamente lo que puede deducirse de sus estudios con Schindler. En los trabajos del entrerriano podemos encontrar una exorbitante cantidad de referencias a la obra helleriana, al menos para lo que era la literatura habitual durante los años cuarenta y cincuenta. Podemos encontrar en su obra menciones a, por lo menos, ocho escritos distintos de Heller, de los cuales solo una parte habían sido traducidos al español al momento de su redacción. ${ }^{1}$ Estas referencias, además de aparecer en las ya mencionadas La crisis del Estado de derecho liberal-burgués e Introducción a la teoría del Estado, se encuentran también en El derecho de resistencia, publicado en 1938; La filosofía del Iluminismo y la Constitución Argentina de 1853, publicado en 1944; Estudios de derecho público. Constitucional, de gentes y eclesiástico que data de 1951; Constitución y pueblo, de 1974 y en el artículo "La filosofía jurídica del artículo 19 de la Constitución Nacional" de 1975. Con esto se constata que el interés del jurista entrerriano por el teórico alemán se mantiene casi a lo largo de toda su producción.

A grandes rasgos, podemos establecer que la recuperación que Sampay hizo de Heller se ordena en torno a cuatro intereses hermenéuticos. El primero de ellos es el fascismo. Principalmente en La crisis del Estado de derecho liberal-burgués, Sampay se sirve del libro de Heller titulado Europa y el fascismo para analizar el régimen fascista en Italia. Este interés, sin embargo, fue dejado de lado rápidamente ya que el jurista entrerriano desarrolló una lectura propia sobre el fascismo y, particularmente, sobre la figura de Benito Mussolini distinta a la que había hecho Heller.

El segundo interés hermenéutico es de carácter jurídico y se centra sobre las nociones de ley y constitución que Heller desarrolló a propósito de la Constitución de

\footnotetext{
1 Nos referimos específicamente a los siguientes escritos: Las ideas políticas contemporáneas [1926], La crisis de la Teoría del Estado [1926], La soberanía [1927], "Democracia y homogeneidad social" [1928], “El concepto de ley en la Constitución del Reich” [1928], “¿Estado de Derecho o dictadura?” [1929], Europa y el fascismo [1931] y Teoría del Estado [1934]. Para el momento en que Sampay escribió y publicó, solo había traducciones españolas de Las ideas políticas contemporáneas, Europa y el fascismo y Teoría del Estado. Sin embargo, el único texto que citó en lengua española fue Europa y el fascismo. El resto, tal como puede verse en sus escritos, fueron citados $-y$, estimamos, leídos- en el original alemán.
} 
Weimar. El primer aspecto que Sampay retoma de la obra helleriana es la distinción entre la ley en su sentido formal y en su sentido material. Estas menciones, que aparecen principalmente en El derecho de resistencia y en "La filosofía jurídica del artículo 19 de la Constitución Nacional", son tomados de una disertación que Heller hizo en el marco de la Asociación de Profesores Alemanes de Derecho Público en 1927 y que se publicó un año más tarde bajo el título "El concepto de ley en la Constitución del Reich" en el boletín de la asociación. El segundo aspecto que retoma Sampay es el concepto de constitución. A lo largo de unas lecciones sobre derecho constitucional que brindó y que se cristalizaron como libro bajo el título Constitución y pueblo en 1973, Sampay retomó el concepto de constitución tal como Heller lo desarrolló en su obra publicada póstumamente en 1934, Teoría del Estado.

El tercer interés hermenéutico que podemos identificar es el del vínculo entre política y ética. En este punto Sampay retoma uno de los aspectos que mayor trascendencia han tenido en la obra de Heller, a saber: los principios jurídicos fundamentales. ${ }^{2}$ Estos principios, que pueden ser de carácter lógico o ético, son grandes lineamientos que, si bien carecen de positividad, guían la interpretación jurídica y constitucional. El desarrollo de estos principios en la obra de Heller puede encontrarse en sus escritos La soberanía de 1927, en el mentado artículo sobre "El concepto de ley en la Constitución del Reich" y en Teoría del Estado. Sampay retoma la noción de principios jurídicos fundamentales en el anexo titulado "El concepto de libertad económica de la Constitución de 1853 y la evolución de la legalidad económica argentina" que incluyó en su obra La filosofía del iluminismo y la Constitución argentina de 1853. Según señala el jurista entrerriano, estos principios dan cuenta del modo en que el Estado, en tanto institución, "sirve a la aplicación y efectividad de los principios éticos por medio del Derecho" (1944: 84) y por tanto cobra sentido su intervención en la economía y su papel regulador.

El último interés con el que Sampay se introduce en la obra de Heller es el de la epistemología y la metodología de la teoría del Estado. En particular, en lo que

2 En buena medida, la noción de "principios jurídicos fundamentales" ha animado la recepción argentina de la obra de Heller en los últimos años. Al respecto, puede verse el artículo "La noción de principios jurídicos en Hermann Heller" de Leticia Vita (2015) y "La legitimidad del poder en la filosofía política de Hermann Heller" de Sergio Raúl Castaño y Andrea Sereni (2016). 
respecta al modo de acceder y conocer la realidad estatal. A nuestro juicio, es aquí donde el entrerriano expone con mayor claridad y sistematicidad lo que, en su consideración, constituyen los alcances y los límites de la concepción helleriana. A su vez, puede observarse con precisión el influjo que aquella causó en Sampay y el modo en que se sirvió de la misma para desarrollar su propia perspectiva. En virtud del volumen de este interés, vamos a dedicarnos aquí a indagar únicamente esta arista de la lectura helleriana de Sampay.

Este artículo se propone, entonces, indagar el papel que Heller desempeña en la obra de Sampay, principalmente en lo relativo a la epistemología y la metodología de la teoría del Estado. Para estos fines, vamos a dividir el trabajo en tres apartados. En el primero, indagamos la recuperación que Sampay hace de Heller a fin de someter a crítica la teoría idealista del Estado, esto es, aquella corriente teórica que consideraba a la estatalidad un ente de carácter abstracto. Rastreamos en él, en un primer subapartado, la exposición que hace en su tesis La crisis del Estado de Derecho liberal-burgués y, posteriormente en un segundo subapartado, los desarrollos que le dedica en Introducción a la Teoría del Estado. Si bien existen corrimientos entre ambos textos, puede identificarse que la obra de Heller funciona como un apoyo para la crítica al idealismo.

En el segundo apartado, en cambio, nos dedicamos a restituir el modo en que Sampay se sirvió de Heller para elaborar su propia concepción de la teoría del Estado, tal como la desarrolla en la segunda parte de Introducción a la teoría del Estado. En este punto, si bien el papel que desempeña la obra de Heller tiene una importancia menor que el que había cumplido en la crítica al idealismo, puede identificarse que Sampay se sirve de Heller para clasificar disciplinarmente a la teoría del Estado y conceptualizar el vínculo entre teoría y política. En el último apartado nos limitamos a recapitular los argumentos y a extraer algunas conclusiones generales.

Antes de iniciar la indagación, debemos mencionar que el vínculo entre Sampay y Heller fue previamente visitado. Si bien no existe ningún artículo que revise 
exclusivamente este vínculo, ${ }^{3}$ sí se dedica una buena cantidad de párrafos en dos de los principales artículos de divulgación que existen sobre la obra del jurista entrerriano, a saber: "Aproximación al pensamiento jurídico y político de Arturo Enrique Sampay" de Juan Fernando Segovia (2007) y "La filosofía política de Arturo E. Sampay" de José Ricardo Pierpauli (2011).

Pueden identificarse en estos artículos tesis opuestas en lo relativo al influjo que Heller causó en Sampay. Mientras que Segovia sostiene que la obra de Heller y, en general, la teoría política y social producida durante la Alemania de Weimar constituye uno de sus principales insumos teóricos, Pierpauli sostiene, en cambio, que el acercamiento que Sampay ensaya a la obra de Heller no tiene otra intención que la de poner en diálogo la "filosofía perenne" aristotélico-tomista con las tendencias teóricas de la época, resultando de poca relevancia el influjo del teórico alemán sobre el jurista enterriano.

\section{La crítica a la teoría idealista del Estado}

a. El conocimiento de la realidad estatal en La crisis del Estado de derecho liberal-burgués

El capítulo inicial de la tesis de Sampay titulada La crisis del Estado de derecho liberal-burgués está dedicado a establecer un "acceso metódico" al problema del Estado, esto es, una serie de lineamientos metodológicos sobre la cuestión de la estatalidad. Allí, resaltan dos grandes aportes que el jurista tomó de la obra de Heller: por un lado, la fundamentación del carácter real del Estado en la actividad humana; por otro, la formulación de conceptos, particularmente la crítica a los tipos ideales y la recuperación de la categoría de "estructura real-histórica" para conceptualizar el conocimiento de la realidad estatal. A lo largo de este subapartado vamos a dedicarnos a exponer ambos desarrollos teóricos.

La fundamentación del carácter real del Estado cobra relevancia a raíz del señalamiento que hace Sampay indicando que una de las principales dificultades a las que se enfrenta el investigador de la teoría del Estado es la existencia de una serie de

3 Sí los hay, por ejemplo, del vínculo entre Arturo Sampay y Carl Schmitt. Al respecto, puede verse Buela (2011), Segovia (2014) y Rodríguez Rial (2016). 
corrientes científicas que conciben a la estatalidad como una estructura de sentido. Esto es, como si se tratara de una entidad abstracta, carente de realidad, y que puede ser definida como "idea, espíritu objetivo, ideología, ordenamiento normativo ideal, abstracción, ficción, o algo por el estilo" (2011a: 48). Esta concepción del Estado como ente abstracto es lo que define a la postura idealista en la teoría del Estado.

A fin de combatir este idealismo, Sampay dedica uno de los apartados del capítulo inicial a desarrollar una de las divisas que informan la Teoría del Estado de Heller, a saber: que el Estado no es un ente abstracto, sino una "forma de vida". Es decir, que es un producto que surge de la actividad sostenida de los seres humanos en tanto constituye "formas", esto es, regularidades en la actividad que pueden ser identificadas bajo las figuras de la legislación, de los procedimientos jurídicos o de las instituciones políticas, entre otros. La actividad humana entendida como "vida" y las regularidades institucionales identificadas como "formas" establecen entre sí una relación dialéctica que Sampay expresa hellerianamente diciendo que el Estado es "vida transformada en forma y forma extraída de la vida" (2011a: 48). ${ }^{4}$ Así, al afirmar que el Estado es una "forma de vida" se expresa, precisamente, esta relación dialéctica entre la vida, entendida como actividad humana, y la forma, entendida como aquellas regularidades de la actividad.

La idea relativa al Estado como forma de vida le interesa a Sampay no únicamente por brindarle, frente al idealismo, un anclaje objetivo enraizándolo en la actividad humana, sino también por dotarlo de estabilidad. Según afirma, la acción del hombre es, por sí misma, inestable y volátil. Ahora bien, en tanto transforma la naturaleza y el medio en el que existe, es capaz de crear objetivaciones que perduren a través del tiempo y trasciendan la acción misma. Estas objetivaciones que pueden ser identificadas bajo la noción de forma, son las que estabilizan el Estado a través del tiempo.

Ahora bien, estas formas no tienen una existencia autónoma respecto de la actividad humana. Más bien, su existencia se apoya en ella. Por lo tanto, si bien en

4 Esta afirmación se trata de una paráfrasis de Teoría del Estado, más precisamente de la afirmación helleriana según la cual "el Estado no es sino una forma de vida humano-social, vida en forma y forma desde la vida" (1971d: 136 [La traducción es nuestra, hay edición al español en Heller (1981: 59)]). 
algunos casos la acción de los seres humanos se orienta a dar conformidad a las formas constituidas y las sostiene, en otros casos la actividad humana, inconforme, se orienta a la reforma. La estabilidad o inestabilidad de las formas, entonces, depende directamente de la conformidad de los seres humanos que las sostienen. Por ello, Sampay sigue a Heller y, tal como hace el teórico alemán, se sirve de la expresión de Renan para describir el vínculo entre la conformidad, la acción y las formas constituidas: "la realidad del Estado solo existe como un plébiscite de tous les jours" (2011a: 48). ${ }^{5}$

La concepción helleriana del Estado como forma de vida, entonces, le brinda herramientas a Sampay para establecer la objetividad y estabilidad de esta formación y disputar, así, frente a la concepción idealista. Ahora bien, no es solo el establecimiento del carácter real del Estado lo que retoma el jurista entrerriano de la obra de Heller, sino también lo que hace al conocimiento del Estado y su representación en conceptos. En otras palabras, le interesa también lo relativo a la formación conceptual. Aquí, una vez más, se ciñe a lo expuesto por Heller en su Teoría del Estado al momento en que critica los conceptos típico-ideales tal como fueron sistematizados por Max Weber y Georg Jellinek.

Según dice Sampay, la formación conceptual en tipos ideales "no puede satisfacer cuando se quiere aprehender la realidad objetiva del Estado" (2011a: 51). Esto significa, según explica, que los tipos ideales, más que representar la realidad estatal, representan las preferencias y los puntos de vista individuales del investigador. Para sostener esta afirmación, Sampay sigue la crítica de Heller a la concepción epistemológica que subyace a los tipos ideales y que se despliega al teorizar el modo en que la realidad se le aparece al sujeto de investigación y al vínculo que se tiende entre ambos.

En primer lugar, se ocupa del modo en que la realidad estatal se le presenta al investigador. En la concepción de Weber y Jellinek, la realidad aparece inmediatamente como un caos ininteligible: al estar compuesta por una variedad de

5 En este pasaje, Sampay omite hacer referencia a Renan y únicamente señala que sigue a Heller. La cita completa es la siguiente: "Así se puede afirmar, con Hermann Heller, que la realidad Estado solo existe como un plébiscite de tous les jours" (2011a: 48). 
afirmaciones y acciones producidas por una multitud de individuos que persiguen finalidades diferentes, la realidad carece de unidad y de inteligibilidad. ${ }^{6}$ En virtud de este carácter con el que se presenta, el investigador necesita ordenar la realidad a fin de comprenderla y conocerla. Por lo tanto, el carácter desordenado de la realidad exige una mediación por parte del sujeto.

La mediación por parte del sujeto se orienta a ordenar intelectualmente la realidad. En virtud de sus interrogantes, intereses de investigación y valores subjetivos, el investigador se ve posibilitado para delimitar y recortar un fragmento de la realidad social, dotarla de inteligibilidad a partir de algún principio teórico que la ordene y representarla en tipos ideales. Los tipos ideales, precisamente, permiten representar una porción de la realidad unilateralizando algunos de sus aspectos y abstrayéndolos, de manera tal que conformen una unidad carente de contradicciones lógicas.

Es en función de estos supuestos que operan a la base de la formación de conceptos típico-ideales que Sampay hace propia la crítica que les había dirigido Heller: según afirma, más que representar la realidad estatal, estos conceptos representan una ficción creada por el investigador en base a sus propias preferencias. En términos de Sampay, los tipos ideales se tratan de una "síntesis pensada subjetivamente", que presenta solamente aquello que fue ordenado y vuelto inteligible por el investigador en función de sus propios intereses y que se "verifica de manera arbitraria" (2011a: 51). Por lo tanto, en función de esta primacía del sujeto en el proceso cognoscitivo y de que el objeto de conocimiento se constituye y representa en función de los intereses y preferencias subjetivas, Sampay decide utilizar el mismo mote que utilizó Heller para denominar a los tipos ideales y la concepción epistemológica que le subyace: "subjetivismo anárquico".

6 La idea de que la realidad se presenta al investigador como un caos puede ser identificada, en la obra de Weber, en el artículo titulado "La 'objetividad' cognoscitiva de la ciencia social y la política social”. Allí señala Weber que, si se pretende conocer la realidad sin presupuestos, solo se arriba a "un caos de 'juicios existenciales' sobre innumerables percepciones individuales" (1904: 52 [La traducción es nuestra; hay edición en español en Weber (2012: 73)]. En Jellinek, en cambio, puede encontrarse esta idea en Teoría general del Estado, al momento en que señala que los fenómenos humanos "muestran una variedad infinita" o bien, que son "infinitamente diversos" (1914: 29 [La traducción es nuestra; hay edición en español en Jellinek (2004: 74-75)]. 
Ahora bien, la crítica al subjetivismo que subyace a la formación conceptual típico-ideal trae aparejada una postulación del vínculo entre investigador y realidad estatal que, una vez más, toma del propio Heller. Es que, según afirma Sampay, "en la superación de este subjetivismo anárquico que aniquila el ser del Estado, tiene la teorética estatal realista de Hermann Heller el carácter de un suceso jalonante" (2011a: 52). Por ello, al subjetivismo anárquico opone, por un lado, la idea de que al investigador la realidad se le presenta como una totalidad ordenada y, por otro, que la formación conceptual adecuada para la realidad estatal no son los tipos ideales, sino los "conceptos-estructura".

En primer lugar, afirma Sampay que el hombre real en ningún momento experimenta la realidad como un caos o una infinidad absoluta. Por el contrario, la realidad se le presenta al investigador como una totalidad estructurada y dotada de significado que comprende casi inmediatamente. Es que, a diferencia de la concepción subjetivista en que el investigador es postulado como una magnitud externa a la realidad, para Sampay la realidad estatal es un "conjunto activo estructurante que lo comprende a sí mismo" (2011a: 52), esto es, que comprende al investigador. En otras palabras, el investigador está inmerso en la realidad estatal y, por lo tanto, tiene una comprensión de ella previa al ejercicio cognoscitivo y a su representación en conceptos científicos. Esto evita, como puede seguirse, que la realidad estatal se le presente al investigador como un caos desordenado.

En segundo lugar, retoma la formación helleriana de "conceptos-estructura" o "estructura real-histórica". Sobre estas, dice que "el propósito malogrado que perseguía la teoría idealista del Estado con los 'tipos ideales', bien se logra con el concepto de las estructuras reales-históricas" (2011a: 52). En estas categorías encuentra Sampay dos aspectos que le permiten postular su superioridad sobre los tipos ideales, a saber: la relación entre lo general y lo particular y la capacidad de aprehender una totalidad compleja.

Sobre el primer aspecto, afirma el jurista que toda estructura tiene un carácter general y un carácter particular. El Estado, por caso, puede ser aproximado a todos los Estados modernos que se dieron sobre el suelo de la cultura occidental judeocristiana. 
Ahora bien, cada Estado cuenta con rasgos individuales que los diferencian del resto de los Estados existentes. Tal como señala Sampay, la noción de estructura realhistórica permite conjugar, estableciendo una escala de abstracción, estos rasgos generales y particulares que tiene un fenómeno histórico como el Estado.

Sobre el segundo aspecto, en cambio, lo que destaca el jurista es la posibilidad que trae el concepto de estructura real-histórica de presentar un ente como una totalidad que no se sigue de sus partes. Esto es, la realidad estatal no es el resultado de la suma de una serie de instituciones, procesos o características, sino que es una totalidad que se presenta como tal. Si bien pueden identificarse aspectos y funciones particulares de la estatalidad, estos no son sino "momentos" que responden a una totalidad unitaria. Al permitir los conceptos-estructura presentar al Estado como una totalidad compuesta de momentos diversos, señala Sampay que bajo esta categoría se evita "representar falsamente a la realidad social como un agregado espacial descomponible en sus partes" (2011a: 53).

En suma, en La crisis del Estado de derecho liberal-burgués, Sampay se sirve de la obra de Heller en dos aspectos centrales de su "acceso metódico" al problema del Estado. Por un lado, en la fundamentación del carácter real de la estatalidad a través de la noción relativa a que el Estado es una "forma de vida", esto es, que involucra la dialéctica entre vida y forma que le da objetividad y estabilidad a la formación estatal. Por otro lado, en la formación de conceptos Sampay sigue a Heller para someter a crítica a los tipos ideales y recuperar la noción de conceptos-estructura que, a su juicio, resultan más adecuados para conceptualizar la realidad estatal y conocer los fenómenos sociales.

\section{b. El problema del "hiato irracional" helleriano en Introducción a la Teoría del}

\section{Estado}

El tratado Introducción a la Teoría del Estado, publicado en 1951, es un trabajo de características distintas a La crisis del Estado de derecho liberal-burgués. Mientras que este último era una tesis en la que se concedían largas citas a los autores en los 


\section{Nicolás Fraile}

que se apoyaba y se restituía sus puntos de vista sin mayores interferencias, en el tratado del 51 el jurista entrerriano adopta una postura mucho más crítica de la literatura que toma como referencia. Allí no solo enjuicia a los autores a través del tamiz aristotélico-tomista, posición filosófica que Sampay asume como propia, sino que arma largas y originales series conceptuales con las que pretende explicar la pérdida de realidad de la teoría del Estado. La obra de Hermann Heller es objeto, precisamente, de una de las colecciones teóricas que Sampay construye y cae, por supuesto, bajo el juicio crítico de la "filosofía perenne".

La primera diferencia que podemos identificar entre el Heller del 43 -esto es, el que describe en La crisis del Estado de derecho liberal-burgués- y el Heller del 51 es su adscripción a la fenomenología. Precisamente, si bien hay menciones previas al teórico alemán, la primera aparición significativa que hace en el tratado de Sampay se da en el capítulo cuarto del libro primero, titulado "El intento fenomenológico de recuperar el objeto real de la teoría del Estado". En él, tras exponer la pérdida de realidad que tuvo el pensamiento moderno y sus consecuencias para la teoría del Estado, pretende dar cuenta de las características generales de la recuperación del ser y la realidad que ensayaron autores como Edmund Husserl, Max Scheler, Theodor Litt y, particularmente, el propio Heller.

Ya en el comienzo del apartado que dedica al teórico alemán, Sampay señala que en el campo de esta disciplina "estuvo a cargo de Hermann Heller la adopción fundamental del método fenomenológico, con los agregados y rectificaciones que veremos" (2011b: 185). Con estos agregados y rectificaciones se refiere, en particular, a la ruptura que hizo frente a Husserl. Tal como puede leerse, Sampay reproduce el juicio de Heller por el cual, al distinguir entre la esencia y la existencia de un fenómeno, la fenomenología husserliana encuentra serias dificultades para regresar a la existencia histórica que tiene la realidad estatal. En palabras del entrerriano, esto se expresa al afirmar que la intuición de esencias husserliana conduce inevitablemente a la "absolutización de una relatividad histórico-social, pues el investigador retendría solo las esencias separadas de la existencia social-histórica que quiere aprehender" (2011b: 186). 
Por ello, indica Sampay, Heller se vio obligado a nutrirse de otros autores que identifica con la tradición fenomenológica: por un lado, recurrió a Hans Freyer y Theodor Litt, a quien Sampay dedicó uno de los apartados del mismo capítulo, para retomar la noción de dialéctica. Por otro lado, señala, se apoyó también en Wilhelm Dilthey y Paul Tillich para desarrollar la gnoseología histórica y tomar la formación relativa a los conceptos-estructura, tal como los vimos en el anterior apartado.

El vínculo entre Heller y Freyer no tiene únicamente carácter anecdótico, sino que se vuelve clave interpretativa de su exposición. Es que, según afirma el entrerriano, uno de los puntos más importantes de la teoría del Estado helleriana es la distinción entre "formaciones de sentido" y "formaciones sociales significativas" que el teórico alemán tomó directamente de Freyer. ${ }^{7}$ Tal como se señala en el texto, una y otra formación refieren a los dos modos de conocimiento que la propia realidad exige. Si cada acto del hombre - y con ello, el conjunto de la realidad social- es "una unidad dialéctica de actividad y sentido" (2011b: 187), las mentadas formaciones son el fundamento desde el cual puede conocerse cada momento dialéctico. Detengámonos con mayor profundidad en esta distinción.

Las formaciones de sentido son las que interesan, valga la redundancia, a las ciencias del sentido. Estas se limitan a comprender el contenido de sentido que resulta inherente a cada acto sin que interfiera la actividad humana, entendiéndolo como una magnitud relevante en virtud de su legalidad y coherencia interna. Tal como explica Sampay, "aíslan el contenido de significación e investigan el conjunto significativo en su legalidad particular, tratando, en lo posible, de evitar todo vínculo con el proceso de real actualización" (2011b: 188). Ejemplos de formaciones de sentido son el derecho o los lenguajes.

Las formaciones sociales, en cambio, refieren al momento de la actividad. Las ciencias que la toman como fundamento son, en la denominación de Freyer, las "ciencias de la realidad". Estas, a diferencia de lo que podría intuirse inmediatamente, no renuncian a aprehender el contenido de sentido de los actos. Por el contrario,

7 Con esto, Sampay hace referencia a que Heller construye su noción de "formación social" [Sozialgebilde] a partir de la concepción de Freyer de la "formación societal" [gesellschaftliche Gebilde], tal como la desarrolla en Sociología como ciencia de la realidad. 


\section{Nicolás Fraile}

pretenden conocer la acción tanto en sus efectos concretos como en el sentido que le es inherente. En términos de Sampay, a estas les interesa conocer "la unidad de acción y sentido, o sea, la realidad social-cultural que es vida espiritualizada" (2011b: 188). Por lo tanto, formaciones sociales son todas aquellas que se apoyan en la actividad humana: el Estado es el ejemplo por excelencia.

La distinción entre formaciones de sentido y formaciones sociales la introduce Heller a raíz de que el idealismo en la teoría del Estado, tal como lo definimos en el apartado anterior, pretende abordar a la formación estatal como si se tratara de una formación de sentido. Esto es, como una magnitud abstracta, relevante en virtud de su legalidad y coherencia interna. Para el teórico alemán, el Estado debía ser abordado como una formación social, atendiendo tanto a su sentido como a los efectos concretos de la actividad humana en la que se apoya. Sampay, que restituye esta distinción, acuerda con la crítica que el alemán realiza al idealismo que pretende leer al Estado como formación de sentido. Sin embargo, se muestra crítico de la noción de formación social y, en general, del ethos que subyace a las ciencias de la realidad.

El puntapié de la crítica de Sampay a las formaciones sociales es la pregunta por la diferencia que existe entre ciencias del sentido y ciencias de la realidad. ¿Qué diferencia hay entre ellas? ¿Se trata meramente de una diferencia cuantitativa, dada por el hecho de que las ciencias de la realidad, además de aprehender el contenido de sentido, permiten aprender el efecto concreto de la actividad humana? O bien, ¿la diferencia que existe entre estos tipos de ciencias es de otra naturaleza? Al responder esta pregunta, el jurista entrerriano afirma que la diferencia entre ciencias del sentido y ciencias de la realidad no es únicamente de carácter cuantitativo. Es decir, no puede estar dada solamente por el hecho de que estas últimas permiten aprehender también las formaciones sociales. Más bien, lo que hay entre estos dos tipos de ciencia es una diferencia que radica en los supuestos que informan a una y otra y está dada por el vínculo que traban entre sí el intelecto y la voluntad.

Sampay, al tratar la diferencia entre las ciencias del sentido y las de la realidad, menciona que las primeras conciben su conocimiento como un "movimiento relativamente autónomo del pensar" (2011b: 188). Las formaciones de sentido, como 
dijimos, son realidades ya constituidas que tienen validez en función de su contenido, legalidad y coherencia interna. Por lo tanto, permiten que el teórico se acerque a ellas con un cierto grado de exterioridad, siendo posible observarlas y conocerlas manteniendo alguna distancia. De allí que no exijan el involucramiento directo del teórico en ellas, sino que su conocimiento puede ser concebido como un ejercicio intelectual.

Las formaciones sociales, en cambio, no se tratan de realidades ya constituidas, sino que están siempre en movimiento y transformación. A su vez, no tienen relevancia únicamente por su contenido y legalidad interna, sino por el vínculo volitivo que se tiende entre ellas y los seres humanos que se hallan a su base. Por último, aquí también opera el supuesto que mencionábamos en el anterior apartado: el investigador forma parte de la realidad estatal. Por lo tanto, no se puede acercar a las formaciones sociales manteniendo distancia o exterioridad. Estos tres elementos, el movimiento de actividad permanente, el vínculo con la voluntad humana y la identidad entre sujeto y objeto, son centrales para fundamentar que el conocimiento de las formaciones sociales no puede involucrar únicamente el intelecto del investigador, sino también su decisión de voluntad. Veamos esto con mayor detenimiento.

Si las formaciones sociales están dadas por la actividad permanente, se sigue que el Estado es un ser dinámico, atravesado por luchas de voluntad intestinas en las que se enfrentan comunidades de voluntad contrapuestas. En virtud de ello, como dice Sampay, "resulta imposible, para el sujeto conocedor, alcanzar un saber que esté desnudo de su querer" (2011b: 190). Es que la realidad estatal, al ser inestable en virtud de las voluntades que se enfrentan en su interior, no puede ser conocida si no se la estabiliza y constituye como un objeto de estudio cerrado. Para lograr esta estabilización, debe mediar una decisión de voluntad del investigador.

La decisión de voluntad del investigador hace las veces de hipótesis que permite el conocimiento. Al estar la realidad estatal en permanente movimiento en virtud de las formaciones de voluntad contrapuestas que actúan a su base, es necesario que el investigador señale a una de estas formaciones como válida y plausible de conformar el futuro del Estado. Tal como afirma Sampay, "en la 


\section{Nicolás Fraile}

contraposición de distintas fuerzas volitivas, se considera a una de ellas como formando el futuro, es decir, como valiosa para él, cualquiera sea su actitud frente a la situación actual" (2011b: 191). Al señalar la validez de una de las formaciones por sobre otra, el investigador prescinde teóricamente del movimiento de la realidad estatal y puede establecer la formación social como objeto de estudio, dispuesto para su conocimiento. Por lo tanto, hay una necesidad teórica en las ciencias de la realidad de que intervenga la voluntad del investigador en el proceso cognoscitivo.

Ahora bien, la intervención de la voluntad no se da únicamente en virtud de esta necesidad teórica. Como decíamos, entre el investigador y el objeto de estudio no hay exterioridad, sino más bien una relación existencial entre ellos por la cual el sujeto forma parte de la realidad estatal. El investigador, al conocer una formación social, conoce también la vida de la que forma parte. Por lo tanto, es imposible que esa decisión de voluntad que señala una tendencia como válida para formar el futuro tenga únicamente carácter de una hipótesis aséptica, sino que habla también del querer del investigador para la realidad en la que existe. Como dice Sampay, el investigador siempre "integra en forma activa la unión social" y lo "atan a ella indestructibles relaciones de ser y querer" (2011b: 188). Por ello, carece de la distancia necesaria para que el objeto se dirija únicamente a su intelecto y se dirige, inevitablemente, "también a su responsable decisión de voluntad" (2011b: 189). La decisión de voluntad interviene en el proceso cognoscitivo, entonces, a raíz del vínculo existencial entre sujeto y objeto.

Si la voluntad interviene en el conocimiento de la realidad estatal en virtud tanto de una exigencia teórica como de la relación existencial que existe entre el investigador y el objeto de estudio, resulta difícil sostener en la teoría helleriana la distinción clásica entre ciencias especulativas y ciencias valorativas. Esto es, entre una ciencia que se dedica a establecer juicios fácticos y una disciplina que establece juicios de valor. Precisamente, la contraposición entre el ser y el deber ser es poco adecuada para la teoría del Estado. Tal como expone Sampay siguiendo a Heller, "la valoración y el conocimiento político realista se hallan, pues, indisolublemente unidos" (2011b: 191). Ahora bien, aquí aparecen sus diferencias: mientras que el teórico alemán 
pretendía sostener la objetividad y la cientificidad de este conocimiento a pesar de la intervención de la voluntad, para Sampay es claro que, bajo la concepción de las ciencias de la realidad, "el conocimiento político queda adherido al antagonismo político" (2011b: 191).

De allí en más, el juicio de Sampay sobre la teoría del Estado de Heller es lapidario. Según afirma, "la fenomenología aplicada a la Teoría del Estado (...) reconduce, al final, a esa misma posición idealista que pretendiera superar" (2011b: 194). De acuerdo con el jurista entrerriano, el idealismo carecía de vínculo con el ser del Estado en virtud de que sus conocimientos eran producto de los puntos de vista arbitrarios del investigador. En el caso de la fenomenología helleriana ocurre algo similar: en lugar de ordenarse la realidad estatal a partir de sus intereses y preferencias, se ordena con la decisión de voluntad del investigador. En otras palabras, Sampay encuentra que los intereses subjetivos del idealismo y la decisión de voluntad helleriana cumplen la misma función: transponer las "vivencias del sujeto conociente a las expresiones del objeto humano conocido" (2011b: 192). Así, no hay conocimiento realista del Estado.

$\mathrm{Si}$, como dice Sampay, el sujeto de conocimiento helleriano describe la realidad del Estado tal como su voluntad se lo dicta, todo el conocimiento que su teorética pueda producir se asienta sobre lo que denomina un hiatus irrationale entre el sujeto y el objeto. Esto es, que no hay ningún vínculo entre uno y otro, sino solamente una decisión de voluntad irracional que suple el ser del Estado. De esta manera, la teorética helleriana carece de algún criterio de veracidad y compone, más bien, "una fabulación que sirve de instrumento de propaganda para las luchas políticas" (2011b: 192).

En suma, si bien en Introducción a la teoría del Estado Sampay vuelve a utilizar a Heller para criticar al idealismo, a diferencia del texto del 43 aquí se produce un alejamiento crítico del teórico alemán. A pesar del tono elogioso que conserva hacia su obra, es lapidario al sostener que su postura se apoya en un hiato irracional entre el sujeto y el objeto que es suturado a través de la voluntad del investigador. En virtud de 


\section{Nicolás Fraile}

ello, el ensayo de un conocimiento realista del Estado que había identificado en Heller termina recayendo en el mismo idealismo que originalmente pretendió superar.

\section{Hacia una Teoría realista del Estado}

La participación de Heller en la Introducción a la Teoría del Estado no termina en la crítica que expusimos en el anterior apartado. Por el contrario, es uno de los pocos autores que aparece también en la segunda parte del tratado titulada "Propedéutica de una Teoría realista del Estado". Si en la primera parte del volumen Sampay pretendía sentar los principales puntos de la crítica a la concepción idealista del Estado, en la segunda pretende establecer los fundamentos sobre los cuales desarrollar una Teoría del Estado realista. Al respecto, hay dos puntos en los que interviene Heller: la clasificación de las ciencias y el esclarecimiento del vínculo entre teoría y política.

En lo que atañe a la clasificación de las ciencias, Sampay dedica a Heller un apartado del capítulo tercero titulado "Sentido con que puede aceptarse la cualificación de la Teoría del Estado como ciencia cultural y ciencia estructural concebida por Hermann Heller". En él expone, precisamente, los argumentos que acercan la teoría realista del Estado a una ciencia de la cultura y, sobre todo, a una ciencia estructural, vinculada a los conceptos-estructura tal como mencionamos previamente.

Sampay coincide con Heller en el hecho relativo a que el ámbito propio de los fenómenos estatales es la cultura. Esto es, que se trata de un producto de la acción humana que introduce fines materiales y espirituales en la naturaleza o, como define sucintamente Sampay, que es una "realidad creada por la eficiencia humana" (2011b: 437). Frente a quienes caracterizan el objeto de la teoría del Estado como propio de la ciencia natural que, incluso, podría ser equiparado con un organismo biológico, el jurista entrerriano afirma que el dominio del Estado es un ámbito que es producto de la acción de los hombres.

Ahora bien, a pesar de la coincidencia entre Sampay y Heller sobre el carácter cultural de la teoría del Estado, hay una diferencia relativa al carácter científico de esta 
disciplina. Es que el jurista entrerriano señala, desde su postura sistemática aristotélico-tomista, que la teoría del Estado no puede ser "una ciencia o filosofía, porque su objeto carece de universalidad y necesidad" (2011b: 438). Al ser el Estado un producto histórico de la eficiencia humana no puede concebirse que su estudio constituya una ciencia. Por ello, señala críticamente, la concepción de ciencia que maneja Heller indica únicamente un "conjunto de conocimientos que presentan un grado suficiente de unidad, de relaciones objetivas y de generalidad, y que es susceptible de llevar a los estudiosos a conclusiones concordantes que se confirman por métodos definidos de verificación" (2011b: 438).

Más allá de esta diferencia, Sampay vuelve a detener su atención en los conceptos-estructura, tal como había hecho en 1943, y en la noción del Estado como estructura histórica en devenir. Sobre los conceptos-estructura, en una caracterización que no difiere demasiado de la que había establecido en aquella obra, sostiene nuevamente que esta formación conceptual permite representar con fidelidad a una comunidad de hombres que persigue un fin común organizada bajo la forma estatal. Sobre la noción de Estado como estructura histórica, adopta esta caracterización de Heller para hacer referencia a una magnitud que no es históricamente estable, sino que varía en virtud de que es un fenómeno cultural, esto es, que es creado y sostenido a través de la incesante actividad de los seres humanos. ${ }^{8}$

El segundo punto por el que Sampay se interesa en la obra de Heller para la constitución de una teoría realista del Estado es el vínculo entre teoría y política. Al respecto, el jurista dedica un apartado en el capítulo cuarto titulado "Relación entre la Teoría del Estado y la política en Hermann Heller". A grandes rasgos, puede apreciarse que este apartado restituye buena parte de los hallazgos desarrollados en el libro primero. Esto es, que para Heller la realidad estatal constituye un objeto que solo puede ser aprehendido si interviene la voluntad del sujeto de conocimiento.

Sobre esto, Sampay coincide en que la realidad estatal se trata de una realidad práctica. Es decir, que es producto de la efectividad humana. Sin embargo, se distancia

8 Si bien la noción del Estado como estructura histórica está identificada con la obra de Heller, particularmente con su Teoría del Estado, cabe decir que Sampay está pensando también en algunos autores como Wilhelm Dilthey, Max Scheler o Rudolf Smend, quienes tenían concepciones similares a la helleriana, al menos en este punto. 
de Heller en el hecho relativo a que no puede haber un conocimiento especulativo de la misma. Es que como vimos, al intervenir la voluntad del investigador en el proceso de conocimiento, el rol del intelecto en la teoría del Estado helleriana queda, a juicio del jurista entrerriano, relegado a un plano secundario y el conocimiento termina apoyándose necesariamente en el querer subjetivo.

Sampay se diferencia al afirmar que el conocimiento de la realidad práctica es siempre especulativo: es la inteligencia del ser humano la que lo conoce en primera instancia. Pero no solamente difiere de Heller en esta apreciación. El entrerriano considera que la valoración de la realidad práctica nunca puede establecerse a partir de la voluntad subjetiva. Por el contrario, debe orientarse por la ciencia política, también denominada política normativa. En otras palabras: en tanto la política tiene como finalidad, según Sampay, procurar el bien común, debe observar una serie de normas derivadas racionalmente del principio por el cual hay que hacer el bien y evitar el mal en la comunidad política, principio fundamental de la política normativa. La alternativa helleriana no hace sino caer en un subjetivismo moral que deja sin realidad la noción de bien común.

En suma, Sampay utiliza la obra de Heller no solo para criticar al idealismo, sino también como un insumo en pos del desarrollo de una Teoría realista del Estado. En primer lugar, identificamos la pertinencia que tienen la clasificación de "ciencia cultural" y la utilización de conceptos-estructura para la concepción de Sampay. En segundo lugar, seguimos la ruptura que realiza frente a Heller en el modo de concebir la relación entre la teoría y la política, anclada principalmente en la disidencia respecto al predominio de la voluntad en la Teoría del Estado y en la política práctica.

\section{Conclusiones}

Este trabajo tuvo como objetivo analizar la lectura que Arturo Sampay hizo de la obra de Hermann Heller, particularmente en lo relativo al acceso y conocimiento de la realidad estatal. Tal como señalamos en la introducción, dividimos el trabajo en dos grandes apartados: por un lado, nos dedicamos a restituir el modo en que se sirve de 
Heller para llevar adelante la crítica a la teoría idealista del Estado, esto es, aquella que lo concibe como una magnitud de orden espiritual o ideal. Por otro lado, analizamos los dos aspectos principales que retoma de Heller para postular su propia concepción de la Teoría del Estado.

Al respecto de la crítica al idealismo, recuperamos en primer lugar su tesis titulada La crisis del Estado de Derecho liberal-burgués, en cuyo apartado metodológico es posible observar que existe un apego significativo a la concepción helleriana. En particular, en lo que hace al carácter de la realidad estatal y a las categorías para conceptualizar el conocimiento.

Sobre el carácter de la realidad estatal, Sampay recupera la dialéctica entre vida y forma que le sirve para dar cuenta que el sostén de los fenómenos políticos y estatales es la actividad humana. Es esta la que, prestando o negando su conformidad, sostiene y transforma los órdenes instituidos. Por ello, no puede sostenerse que el Estado se trate de un ente espiritual o ideal, sino que tiene una expresión concreta y objetiva en la cultura.

Sobre la categoría "estructura real-histórica", podemos mencionar primeramente que su introducción viene antecedida por la crítica a la concepción que subyace a los tipos ideales. Siguiendo a Heller, Sampay señala que el conocimiento que estos brindan no es sino una "síntesis subjetiva mental" que hace el investigador en virtud de sus propios puntos de vista y que se apoya, primordialmente, en la noción de que la realidad social se presenta como un caos informe y que existe una separación tajante entre sujeto y objeto. Precisamente, tras señalar que dicha separación no existe y que la realidad siempre resulta un orden estructurado para el investigador, introduce la categoría de "estructura real-histórica".

La crítica al idealismo también informa la recepción que hace de Heller en Introducción a la Teoría del Estado. En este texto no solamente se sirve de la obra del teórico alemán, sino que además lo enmarca al interior de la corriente fenomenológica. Tal como señalamos, uno de los propósitos de los teóricos que Sampay introduce al interior de esta corriente es el de recuperar el ser del Estado. Precisamente, Heller aparece como la máxima expresión de este objetivo y como 
quien más lejos llegó con el mismo. Sin embargo, Sampay identifica algunas dificultades en la concepción helleriana que lo llevan, a diferencia de lo que ocurría en su obra de 1943, a tomar distancia del teórico alemán.

Tras retomar la distinción entre ciencias del sentido y ciencias de la realidad, Sampay observa que la principal diferencia entre una y otra estriba en el papel que juegan la voluntad y el intelecto en una y otra. Mientras que las ciencias del sentido rechazan la intervención de la voluntad pues fundamentan el conocimiento únicamente en el intelecto, las ciencias de la realidad aceptan que la voluntad interviene en ellas, en virtud de la relación existencial entre sujeto y objeto y del carácter de estructura en devenir que tiene el Estado.

Ahora bien, lo que identifica Sampay es que, a pesar de la pretensión realista que tienen estas últimas, terminan recayendo en una postura similar al idealismo. Las ciencias de la realidad se apoyan sobre un hiato irracional que existe entre sujeto y objeto y que es subsanado por la voluntad del investigador. En otras palabras, en virtud del papel que juega la voluntad en el proceso de conocimiento, el sujeto termina por describir la realidad no de acuerdo con cómo es esta, sino de acuerdo con lo que su voluntad le dicta. De esta manera, el objetivo de recuperar la realidad para la teoría del Estado se malogra y Sampay, a diferencia del texto de 1943, se aleja de los postulados hellerianos.

Este alejamiento, sin embargo, no significó que Heller fuera dejado completamente de lado para la formulación de una Teoría realista del Estado. En la segunda parte del tratado de 1951, en el que pretende establecer los fundamentos de una teoría con dichas características, la figura del teórico alemán vuelve a aparecer vinculada a la clasificación de las ciencias y a la relación entre teoría y política. Sobre la clasificación de las ciencias, Sampay encuentra un gran acierto en la caracterización de la Teoría del Estado como ciencia cultural y como ciencia de estructuras, a pesar del desacuerdo fundamental que tiene sobre la noción de ciencia. Sobre la relación entre teoría y política, el papel que tiene Heller es más bien residual y solo le sirve a Sampay para introducir sus propias apreciaciones, sin que exista una recuperación de lo dicho por Heller para su conceptualización. 
En líneas generales, puede decirse que el papel que la obra de Heller tiene para Sampay es más bien el de un eficaz arsenal crítico del idealismo teórico-estatal. Sus apariciones más significativas y los comentarios más elogiosos que hace el jurista entrerriano sobre Heller son, precisamente, a raíz de sus críticas a Jellinek y Weber, principalmente. Si bien, como vimos, también cumple un papel en la formulación de la teoría del Estado que Sampay lleva adelante, el lugar que ocupa es más bien secundario y se ve fuertemente opacado por los teóricos de ascendencia aristotélicotomista, en quienes Sampay se apoya para desarrollar los fundamentos teóricos de su concepción. Para este aspecto propositivo de su obra, resulta pertinente la hipótesis de Pierpauli (2011), relativa a que el acercamiento de Sampay a la teoría del Estado. 


\section{Nicolás Fraile}

\section{Bibliografía}

Buela, A. (2011). Sampay y Schmitt: una relación crítica. Metapolítica I. Lo nacional en la nueva estrategia suramericana. Buenos Aires: Editorial Docencia.

Castaño, S. y Sereni, A. (2016). La legitimidad del poder en la filosofía política de Hermann Heller". Philosophia, 76 (1), 9-35.

Gómez Arboleya, E. (1963). La teoría del derecho internacional en el pensamiento de Hermann Heller. Estudios de teoría de la sociedad y del Estado (pp. 126-183). Madrid: Centro de Estudios Constitucionales.

Heller, H. (1971). Gesammelte Schriften, III. Leiden: A. W. Sijthoff.

Heller, H. (1981). Teoría del Estado. México: Fondo de Cultura Económica.

Jacobson, A. y Schlink, B. (2001). Weimar: A Jurisprudence of Crisis. California: The University of California Press.

Jellinek, W. (1914). Allgemeine Staatslehre. Berlin: O. Häring.

Jellinek, W. (2004). Teoría general del Estado. México: Fondo de Cultura Económica.

Llanque, M. (2019). Hermann Heller and the Republicanism of the Left in the Weimar Republic. Jus Politicum, 23, 13-30.

Mommsen, H. (1962). Zur Verhältnis von politischer Wissenschaft und Geschichtswissenschaft in Deutschland. Vierteljahrshefte für Zeitgeschichte, 10 (4), 541-572.

Pierpauli, José Ricardo (2011). La filosofía política de Arturo E. Sampay. En Sampay, A. Introducción a la Teoría del Estado (pp. XVII-LXXXII). Buenos Aires: Editorial Docencia.

Rodríguez Rial, G. (2016). Carl Schmitt y el Estado de Derecho como forma política. Un diálogo con Arturo Sampay y Norberto Bobbio. Revista de Derecho y Humanidades, 28, 13-39.

Sampay, A. (1944). La filosofía del Iluminismo y la Constitución argentina de 1853. Buenos Aires: Depalma.

Sampay, A. (2011a). La crisis del Estado de Derecho liberal-burgués. Buenos Aires: Editorial Docencia.

Sampay, A. (2011b). Introducción a la Teoría del Estado. Buenos Aires: Editorial Docencia. 
Segovia, J. (2007). Aproximación al pensamiento jurídico y político de Arturo Enrique Sampay. Catolicismo, peronismo y socialismo argentinos. Anales de la Fundación Francisco Elías de Tejada, 13, 165-192.

Segovia, J. (2014). El derecho entre iusnaturalismo, decisionismo y personalismo. Arturo Sampay lector de Carl Schmitt. Anales de la Fundación Francisco Elías de Tejada, 20, 133-156.

Vita, L. (2015). La noción de principios jurídicos en Hermann Heller. Isonomía, 43, 49-75.

Weber, M. (1904). Die "Objektivität" sozialwissenschaftlicher und sozialpolitischer Erkenntnis. Archiv für Sozialwissenschaft und Sozialpolitik, 19 (1), 2287.

Weber, M. (2012). La "objetividad" de la ciencia social y la política social. Ensayos sobre metodología sociológica (pp. 43-109). Buenos Aires: Amorrortu Editores. 